\title{
Investigation of pathogenic mechanisms in multiple colorectal adenoma patients without germline APC or MYH/MUTYH
} mutations

\section{Thirlwell', KM Howarth', S Segditsas', G Guerra', HJW Thomas'2, RKS Phillips², IC Talbot ${ }^{2}$, M Gorman', MR Novelli', OM Sieber' and IPM Tomlinson ${ }^{*}, 1,4$}

'Molecular and Population Genetics Laboratory, London Research Institute, Cancer Research UK, 44, Lincoln's Inn Fields, London WC2A 3PX, UK; ${ }^{2}$ Cancer Research UK Colorectal Cancer Unit, St Mark's Hospital, Watford Road, Harrow HAI 3UJ, UK; ${ }^{3}$ Department of Histopathology, Rockefeller Building, University College London, London WCIE 6BT, UK; ${ }^{4}$ Institute of Cancer, Bart's and the London Medical School, Queen Mary College, London, UK

Patients with multiple $(5-100)$ colorectal adenomas (MCRAs) often have no germline mutation in known predisposition genes, but probably have a genetic origin. We collected a set of 25 MCRA patients with no detectable germline mutation in APC, MYHIMUTYH or the mismatch repair genes. Extracolonic tumours were absent in these cases. No vertical transmission of the MCRA phenotype was found. Based on the precedent of $\mathrm{MYH}$-associated polyposis (MAP), we searched for a mutational signature in 24I adenomatous polyps from our MCRA cases. Somatic mutation frequencies and spectra at APC, K-ras and BRAF were, however, similar to those in sporadic colorectal adenomas. Our data suggest that the genetic pathway of tumorigenesis in the MCRA patients' tumours is very similar to the classical pathway in sporadic adenomas. In sharp contrast to MAP tumours, we did not find evidence of a specific mutational signature in any individual patient or in the overall set of MCRA cases. These results suggest that hypermutation of APC does not cause our patients' disease and strongly suggests that MAP is not a paradigm for the remaining MCRA patients. Our MCRA patients' colons showed no evidence of microadenomas, unlike in MAP and familial adenomatous polyposis (FAP). However, nuclear $\beta$-catenin expression was significantly greater in MCRA patients' tumours than in sporadic adenomas. We suggest that, at least in some cases, the MCRA phenotype results from germline variation that acts subsequent to tumour initiation, perhaps by causing more rapid or more likely progression from microadenoma to macroadenoma.

British Journal of Cancer (2007) 96, I729-1734. doi:I0.1038/sj.bjc.6603789 www.bjcancer.com

Published online 15 May 2007

(c) 2007 Cancer Research UK

Keywords: multiple colorectal adenoma; APC mutations; MYH/MUTYH mutations

Individuals who develop multiple colorectal adenomas (MCRAs) are a genetically and clinically heterogeneous group who are at increased risk of colorectal cancer (CRC). The MCRA phenotype can usefully, but arbitrarily, be defined as 5-100 adenomas during an individual's lifetime. Germline mutations in the $A P C$ gene account for about $15 \%$ of cases with an MCRA phenotype and such patients are classified as having the dominantly inherited condition, attenuated FAP (AFAP) (Spirio et al, 1999). An additional $\sim 35 \%$ of such patients have biallelic germline mutations in the MutY-homologue (MUTYH, MYH) gene and are classified as having the recessive condition $\mathrm{MYH}$-associated polyposis (MAP) (Sampson et al, 2003; Sieber et al, 2003). MYH deficiency causes colorectal adenomas through somatic hypermutation of the APC and K-ras genes. The remaining MCRA patients have no known underlying cause for their phenotype.

\footnotetext{
*Correspondence: Professor IPM Tomlinson;

E-mail: ian.tomlinson@cancer.org.uk

Received 20 February 2007; revised 13 April 2007; accepted 18 April

2007; published online 15 May 2007
}

MYH was found to be a MCRA predisposition gene because a striking excess of somatic $\mathrm{G}: \mathrm{C}>\mathrm{T}: \mathrm{A}$ transversion was observed incidentally during an analysis of the APC gene (Al-Tassan et al, 2002). A similar spectrum of K-ras mutations is also found in MAP patients (Lipton et al, 2003a). In another Mendelian bowel tumour syndrome, hereditary nonpolyposis colon cancer (HNPCC), the effects of DNA mismatch repair (MMR) deficiency can be detected somatically through the presence of microsatellite instability (MSI) and frameshift mutations in short repeats within genes such as $B A X$ and TGFBR2 (Woodford-Richens et al, 2001). It follows that, should some of the remaining MCRA cases be caused by a defect in DNA repair or by some other form of hypermutation, the somatic mutation spectrum will reflect the underlying cause of genomic instability.

The primary tumour predisposition in familial adenomatous polyposis (FAP) and MAP is to colorectal adenomas via $A P C$ mutation. FAP and MAP patients develop not only macroscopic adenomatous polyps, but also microadenomas (each comprising at most a few dysplastic crypts) within the large bowel (Lipton et al, 2003a). This is consistent with an increased rate of adenoma initiation in FAP and MAP. In HNPCC, by contrast, the primary predisposition is probably to carcinoma (or progression from adenoma to carcinoma) and microadenomas are generally absent. 
The presence or absence of microadenomas in MCRA patients of unknown origins might therefore provide clues about the origins of the disease. A similar line of reasoning can be applied to the observation that (A)FAP and MAP patients frequently develop extracolonic adenomas, especially in the duodenum. The similarity of the MAP and (A)FAP phenotypes suggests that all individuals predisposed to inactivation of APC may develop both colorectal and duodenal adenomas. Interestingly, HNPCC patients are at greatly increased relative risk - but low absolute risk - of small bowel cancer, but have very few adenomas at this site.

To address the origins of the MCRA phenotype, we have determined the spectrum of somatic mutations at $A P C$, K-ras and $B R A F$ in tumours from 25 MCRA patients with no detectable germline mutation in $A P C, M Y H$ or the MMR genes. We have supplemented our molecular analysis with phenotypic data, microadenoma counts and immunohistochemistry for $\beta$-catenin expression. We have compared our results with data from MAP and (A)FAP patients. Our results help to classify the remaining MCRA cases and provide clues to their genetic origins.

\section{MATERIALS AND METHODS}

\section{Sample collection and preparation}

Twenty-five MCRA patients were recruited through St Mark's Hospital Colorectal Cancer Unit and blood was routinely sampled. Informed consent was obtained before sample collection. Inclusion criteria for the study were between 10 and 100 synchronous or metachronous colorectal adenomas, irrespective of follow-up or (prophylactic) colectomy (see Table 1 for clinical information). A total of 241 formalin-fixed, paraffin-embedded adenomas were collected. Germline mutations in APC and $M Y H$ had been excluded by screening all exons and exon-intron boundaries using fluorescence single-stranded conformational polymorphism (FSSCP) analysis or denaturing HPLC analysis (details available from authors). In addition, multiplex ligation-dependent probe amplification (MLPA) analysis had been used to screen for germline deletions or duplications of APC using the Salsa MLPA kit P043 APC (MRC Holland, Amsterdam, The Netherlands) according to the manufacturer's instructions. Germline mutations in the MMR genes $M S H 2, M L H 1$ and $M S H 6$ had been excluded previously using F-SSCP analysis (data not shown). In support of these findings, no MSI was observed in our patients' adenomas at the markers used for loss of heterozygosity (LOH) analysis (see LOH analysis) and we therefore did not undertake MLPA analysis for the MMR loci. We also collected 56 formalin-fixed, paraffin-embedded sporadic adenomas (patients with one or two adenomas and no reported family history) from 29 individuals. Existing sets of adenomas from AFAP and MAP patients were largely available for comparison (Lamlum et al, 2000; Sieber et al, 2003, 2006) although a small amount of extra data was collected for this study. For all patients, DNA was extracted from peripheral blood using standard methods. Tumour and normal tissues were dissected from sections cut from archival, paraffin-embedded specimens and we estimated that the resulting tumour DNAs were derived from at least $70 \%$ dysplastic cells. DNA was extracted from these by standard proteinase $\mathrm{K}$ digestion at $55^{\circ} \mathrm{C}$.

Table I Clinical details and summary of somatic APC mutations in MCRA patients

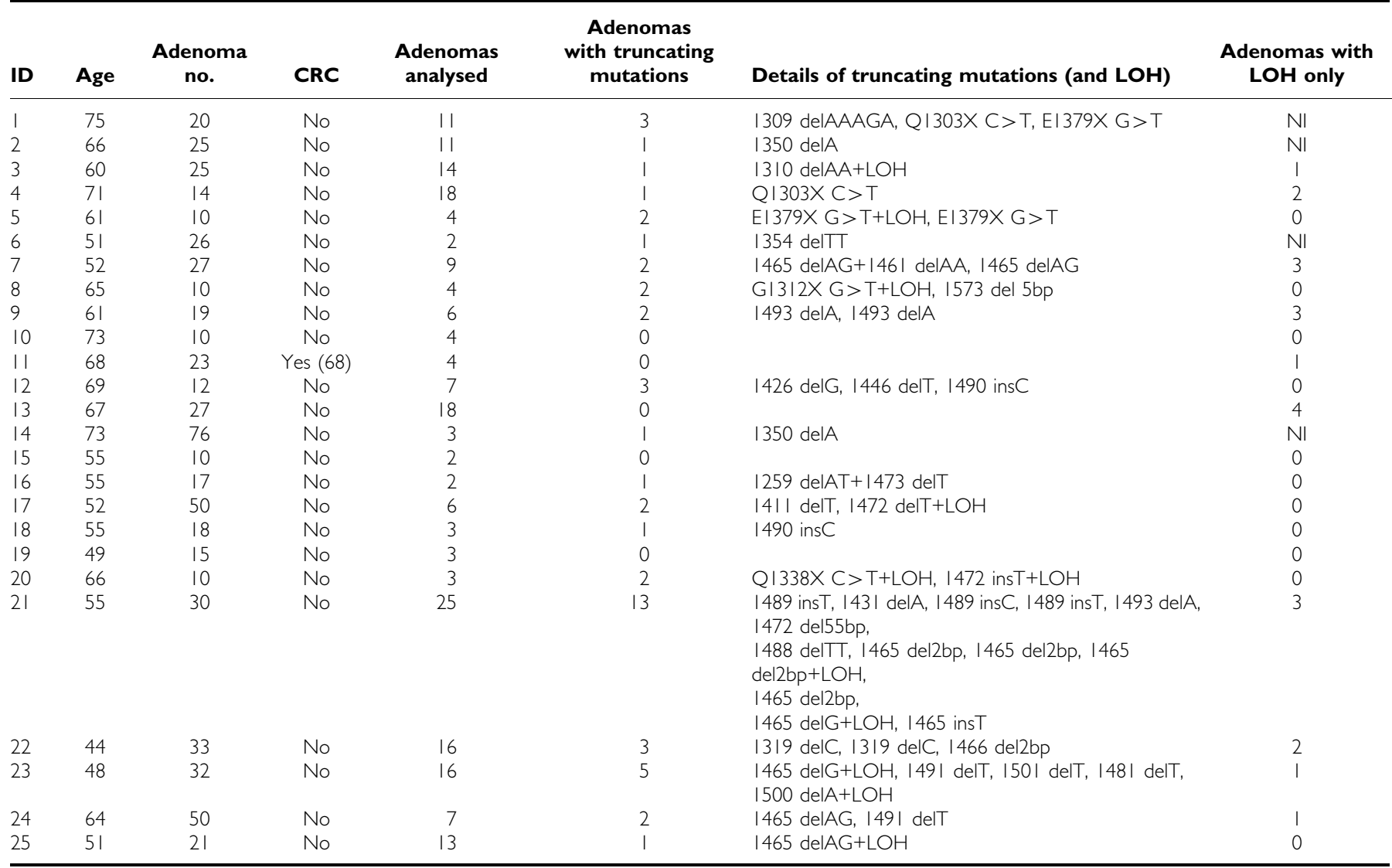

Abbreviations: $\mathrm{CRC}=$ colorectal cancer; $\mathrm{LOH}=$ loss of heterozygosity; $\mathrm{MCRA}=$ multiple colorectal adenoma; $\mathrm{NI}=$ not informative. The table shows: age at diagnosis (years), total number of adenomas developed to time of study, presence of colorectal carcinoma (and age), number of adenomas analysed in study' number of adenomas with any somatic truncating APC mutation found, details of mutations found (codon plus specific base change) including LOH if present and number of adenomas with LOH but no truncating mutation found ( $\mathrm{NI}$ at all markers). 


\section{Mutation detection}

F-SSCP analysis was used to screen formalin-fixed, paraffinembedded tumours for somatic APC mutations between codons 1220 and 1620 , approximately corresponding to the mutation cluster region and covering the region between the first 20 amino acid $\beta$-catenin binding/degradation repeat (20AAR) and the first SAMP repeat. The region was subdivided into 12 amplicons (details available from authors). Any sample with a bandshift was sequenced directly in both orientations from a new PCR product. Exon 1 of $\mathrm{K}$-ras, exon 3 of $\beta$-catenin and the mutational hotspot at codon 600 of BRAF were screened by direct DNA sequencing in both orientations. Novel mutations were identified through comparison with the COSMIC database (http://www.sanger.ac.uk/ genetics/CGP/cosmic) and the European APC database (LaurentPuig et al, 1998).

\section{LOH analysis}

$\mathrm{LOH}$ at $A P C$ was analysed using microsatellite markers just distal to the locus (D5S346, D5S421 and D5S656) on the ABI 3100 sequencer. Constitutionally homozygous markers (and occasional PCR failures) were scored as noninformative. At each marker, $\mathrm{LOH}$ was considered present if the area under one allelic peak in the adenoma was less than 0.5 times or greater than two times that of the other allele, after correcting for the relative allelic areas using constitutional DNA. In the infrequent event of discordance between markers, precedence was given to the marker closest to the $A P C$ gene.

\section{Immunohistochemistry}

Paraffin-embedded sections $(4 \mu \mathrm{m})$ were dewaxed and rehydrated using a standard xylene method. Endogenous peroxidase was blocked by incubating the slides in $0.3 \%$ hydrogen peroxide $\left(\mathrm{H}_{2} \mathrm{O}_{2}\right.$; BDH Chemicals Ltd., Poole, Dorset, UK)/phosphate-buffered saline (PBS) for $30 \mathrm{~min}$. The slides were incubated in normal rabbit serum (NGS-1:25; DAKO Ltd., Ely, Cambridgeshire, UK) for 10 min to block nonspecific binding. Primary antibody ( $\beta$-catenin; Transduction Labs, BD Biosciences, Oxford, UK) was added to the slides at 1:100 dilution and incubated for $45 \mathrm{~min}$. Positive and negative control slides were included in every run. The slides were then washed in PBS and incubated with secondary antibody (biotinylated rabbit anti-mouse Ig; DAKO Ltd.) for $35 \mathrm{~min}$. The slides were incubated for $30 \mathrm{~min}$ at room temperature, washed in PBS and then incubated with streptavidin-peroxidase conjugate (1:500, P397; DAKO Ltd.) for $35 \mathrm{~min}$ at room temperature. The peroxidase activity was demonstrated by activated 3,3'-diaminobenzidinetetrahydrochloride solution (Sigma D5637, Poole, Dorset, UK) and $0.1 \% \mathrm{H}_{2} \mathrm{O}_{2}$. The slides were counterstained in Cole's haematoxylin (Pioneer Research Chemicals Ltd., Colchester, Essex, UK), dehydrated and mounted using Pertex mountant (CellPath, Newton, Powys, UK). Immunohistochemistry was performed on the multiple adenoma group, sporadic adenomas and, for comparison, a set of patients with FAP. Slides were then examined using a conventional light microscope and the level of expression of $\beta$-catenin $(1,2$ or 3$)$ was recorded separately for the nucleus, cytoplasm and cell membrane according to the intensity of staining in five random high-powered fields per slide. This was compared directly with staining in normal crypts from each sample. For membranous expression, in order to normalise results across different runs of immunohistochemistry, the abnormal staining for each slide was divided by the membrane staining in normal cells. Normal cytoplasmic and nuclear expression was so weak that such a correction was not required. Immunohistochemistry was scored by $\mathrm{CT}$ and $\mathrm{KH}$, under the guidance of a gastrointestinal histopathology specialist $(\mathrm{MN})$; any differences were resolved by consensus.

\section{RESULTS AND DISCUSSION}

\section{Features of the MCRA patients}

The clinical features of the 25 MCRA patients are shown in Table 1. All were of white UK origin. The mean age at presentation was 60 years $($ median $=61$, range $=44-70)$. Mean polyp number at presentation was 24 (median $=21$, range $=10-76$ ) and the great majority of polyps were mildly dysplastic tubular or tubulovillous adenomas less than $5 \mathrm{~mm}$ in diameter. One patient had CRC at the time of presentation. No patient had upper gastrointestinal adenomas and there were no other notable or consistent extracolonic features. Most MCRA patients were isolated cases, although a few patients had a single sibling affected with multiple adenomas. In no case was there multigenerational inheritance of CRAs. In five patients, CRCs were present in other family members, although none of these individuals had been reported as having adenomas.

Our MAP patients presented at a median age of 57 years (range $=54-69$ ), not significantly different from the MCRA patients $(P=0.476$, Wilcoxon test). The AFAP patients (median age $=43$ years, range $=27-68$ ) were significantly younger than the MCRA group ( $P=0.027$, Wilcoxon test). We did not undertake a comparison of polyp numbers among the patient groups, since this was determined by our inclusion criteria, but the presence of CRC at presentation was similarly frequent in all three patient types (details not shown).

The family histories and phenotypes of our MCRA cases therefore resembled MAP, except that we found no evidence of a predisposition to extracolonic disease in the MCRA group. If the MCRA cases had, like MAP patients, a tendency to hypermutation of $A P C$ in the gastrointestinal tract, we might expect a similar spectrum of disease to MAP or (A)FAP. Therefore, it is possible that MCRA results either from hypermutation of $A P C$ in the large bowel only or from a distinct pathogenic mechanism that does not involve the tendency to inactivate $A P C$.

\section{Somatic mutation and LOH frequencies are similar in MCRA and sporadic adenomas, but different from AFAP and MAP}

We detected truncating, somatic APC mutations in 49 of $221(22 \%)$ adenomas from the MCRA group (Table 1). Of 190 informative tumours, $32(17 \%)$ showed allelic loss (LOH). Two truncating

Table 2 Comparison between APC mutation frequencies in MCRA and other patients

\begin{tabular}{|c|c|c|c|c|}
\hline & MCRA & Sporadic & AFAP & MAP \\
\hline $\begin{array}{l}\text { Truncating mutation } \\
\mathrm{LOH}\end{array}$ & $\begin{array}{l}49 / 221(22 \%) \\
32 / 190(17 \%)\end{array}$ & $\begin{array}{c}10 / 56(18 \%)^{\mathrm{a}} \\
5 / 56(9 \%)^{\mathrm{d}}\end{array}$ & $\begin{array}{l}91 / 242(38 \%)^{b} \\
19 / 230(8 \%)^{e}\end{array}$ & $\begin{array}{c}25 / 34(74 \%)^{c} \\
1 / 34(3 \%)^{f}\end{array}$ \\
\hline
\end{tabular}

Abbreviations: AFAP = attenuated familial adenomatous polyposis; MAP = MYH-associated polyposis; MCRA = multiple colorectal adenoma. Number of adenomas with mutations out of total successfully analysed (\%) are shown, as are numbers of adenomas with LOH at APC out of total informative tumours (\%). $P$ values for comparison of mutation and $\mathrm{LOH}$ frequencies with those in the MCRA group using Fisher's exact test are as follows: ${ }^{\mathrm{a}} P=0.59 ;{ }^{\mathrm{b}} P<0.00 \mathrm{I} ;{ }^{\mathrm{c}} P<0.00 \mathrm{I} ;{ }^{\mathrm{d}} P=0.20 ;{ }^{\mathrm{e}} P=0.0 \mathrm{I} ;{ }^{\mathrm{f}} P=0.022$. Data were derived from this study, supplemented by our published work on MAP patients (Lipton et al, 2003a) and AFAP (Sieber et al, 2006). 
somatic APC changes were detected in two tumours and 11 tumours had a single $A P C$ mutation and $\mathrm{LOH}$. The APC mutation and $\mathrm{LOH}$ frequencies were not significantly different from those found in the sporadic adenomas (Table 2); however, the AFAP and MAP tumours had significantly higher frequencies of truncating mutations and lower frequencies of LOH than the MCRA tumours (Table 2). No adenoma was found to have a $\beta$-catenin mutation.

A total of $12(12 \%) \mathrm{K}$-ras mutations were detected in 97 MCRA tumours analysed; 10 mutations occurred at codon 12 and two mutations at codon 13. All of the changes - G12A, G12D, G12V and G13D - had been reported previously to occur frequently in sporadic adenomas and CRCs. A little surprisingly, nine out of $85(11 \%)$ MCRA adenomas analysed had a BRAF mutation (V600E); none of these adenomas had serrated morphology. No tumour harboured both K-ras and BRAF mutations. The K-ras and BRAF mutation frequencies in the MCRA tumours were not significantly different from those in the sporadic adenomas (five out of $34(P=0.47)$ and five out of $40(P=0.73)$, respectively; Fisher's exact test).

The similar frequencies of changes at APC, K-ras and BRAF in MCRA and sporadic cases suggest that the genetic pathways of tumorigenesis overlap, at least as regards these three genes. The differences from MAP and AFAP reinforce the possibility that hypermutation of $A P C$ and/or K-ras does not drive tumorigenesis in MCRA patients.

\section{No definitive mutational signature in the MCRA patients' tumours}

In the MCRA patients' tumours, the total of 51 truncating APC mutations (Table 1) comprised 44 frameshifts (small insertions or deletions) and seven nonsense mutations ( $3 \mathrm{C}: \mathrm{G}>\mathrm{T}: \mathrm{A}$ transitions and four $\mathrm{G}: \mathrm{C}>\mathrm{T}: \mathrm{A}$ transversions). Although the proportion of frameshift mutations was higher than in the APC mutation database (Laurent-Puig et al, 1998), this probably resulted from detection bias in small, archival tumours, which were the only samples available from our patients. We have previously observed decreased mutation detection sensitivity in such samples (Groves et al, 2002). In support of this contention, the excess of frameshifts in the MCRA tumours was very similar $(P=0.44$, Fisher's exact test) to that in our sporadic adenomas, which had acquired eight frameshift changes plus one $\mathrm{A}: \mathrm{T}>\mathrm{T}: \mathrm{A}$ transition and one $\mathrm{G}: \mathrm{C}>\mathrm{T}: \mathrm{A}$ transversion. The APC mutation spectrum in the MCRA tumours contrasted with the finding that all 25 truncating mutations in the MAP tumours were $\mathrm{G}: \mathrm{C}>\mathrm{T}: \mathrm{A}$ transversions $(P<0.001$, Fisher's exact test).

We then considered whether there was evidence of a 'mutational signature', especially whether individual patients had a tendency to frameshift changes, to specific nonsense mutations or to $\mathrm{LOH}$ at $A P C$. In general, no such tendency was evident (Table 1). However, one patient (no. 21) had acquired frameshift mutations in 13 tumours and no nonsense changes and another (no. 23) had five frameshift changes and no nonsense changes. These frequencies were not significantly different from those expected $(P>0.1$, exact binomial test). It was, however, striking that all changes in the tumours of patient nos. 21 and 23 occurred in a particular region of the APC gene, between the second and third 20 amino-acid repeats that are involved in $\beta$-catenin degradation. Such a spectrum of mutations might be expected in patients with a germline APC mutation before codon 1280 (Lamlum et al, 1999; Albuquerque et al, 2002; Crabtree et al, 2003) or with a deficiency in MMR that did not manifest as MSI in adenomas (Wu et al, 1999). For these reasons, we rescreened patient nos. 21 and 23 for germline mutations throughout the $A P C$ and the MMR genes $M L H 1, M S H 2, M S H 6$ and PMS2. We included normal tissue from around the adenomas in this screen in case the cases were mosaics. However, no pathogenic changes were found in any of the genes analysed.

In the MCRA patients, the K-ras mutations comprised six $\mathrm{G}: \mathrm{C}>\mathrm{A}: \mathrm{T}$, five G:C: $>\mathrm{T}: \mathrm{A}$ and one G:C $>\mathrm{C}: \mathrm{G}$ changes. Overall, this mutation spectrum was similar to that of the sporadic adenomas, which had nine $\mathrm{G}: \mathrm{C}>\mathrm{A}: \mathrm{T}$, three $\mathrm{G}: \mathrm{C}>\mathrm{T}: \mathrm{A}$ and two $\mathrm{T}: \mathrm{A}>\mathrm{A}: \mathrm{T}$ mutations $(P=0.31$, Fisher's exact test). Similar to $A P C$, these K-ras mutation spectra contrasted with the sole change, $\mathrm{G} 12 \mathrm{C}$ (G:C> T:A), found in MAP tumours. As expected, all of the $B R A F$ mutations were $\mathrm{T}: \mathrm{A}>\mathrm{A}: \mathrm{T}$ transitions resulting in the $\mathrm{V} 600 \mathrm{E}$ change. No adenoma had both K-ras and BRAF mutations. There
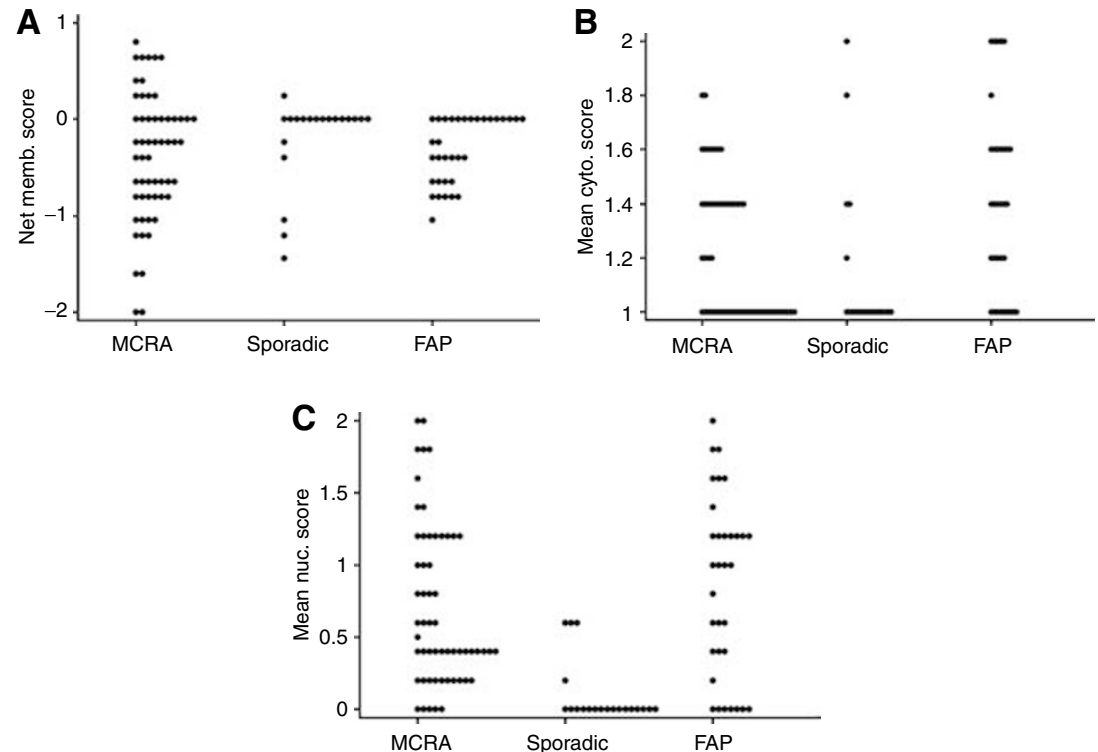

Figure I $\quad \beta$-Catenin expression in adenoma from MCRA and sporadic patients and FAP cases. The dot plots show $(\mathbf{A})$ the net mean membranous score, (B) the mean cytoplasmic score and $(\mathbf{C})$ the mean nuclear score from $\beta$-catenin immunohistochemistry for MCRA adenomas (series I), sporadic adenomas (series 2) and FAP adenomas (series 3). Note that, for ease of depiction, the mean scores from individual adenomas are shown as calculated to one decimal place, although for statistical analysis, only mean integer values were used. The plots clearly show the lower nuclear expression in sporadic than MCRA and FAP adenomas. Ideally, a higher mutation pick up rate with less detection bias could be achieved in a larger series of patients resulting in the analysis of a higher number of adenomas. 
was no association between the presence of $A P C$ and K-ras or $B R A F$ mutations (details not shown).

Overall, these findings strongly suggested that there is no common mutational signature in the MCRA group of patients subject to the limitations of screening large genes, such as APC in DNA of relatively poor quantity and quality. Despite these difficulties, the difference from MAP was striking, once again suggesting that there is no specific tendency for hypermutation in our MCRA cases.

\section{No colorectal microadenomas in MCRA cases}

Haematoxylin an eosin-stained sections of large bowel from FAP, AFAP, MAP and multiple adenoma patients were examined by a histopathology specialist (MN) for the presence of microadenomas (dysplastic lesions $<1 \mathrm{~mm}$ diameter) in otherwise normal large bowel mucosa. Microadenomas were present in 16 out of 19 FAP sections examined, the mean density being $0.22 / \mathrm{mm}$. In AFAP, microadenomas were present in two out of seven sections examined from four patients, with mean density of $0.03 / \mathrm{mm}$. In MAP, microadenomas were present in three out of 16 sections examined from six patients at $0.01 / \mathrm{mm}$. No microadenomas were found on examination of 16 sections from seven MCRA patients. The majority of specimens examined were from colectomies and therefore sporadic adenoma cases could not be assessed, as it is very unusual for colectomy to be undertaken in these patients.

Historically, microadenomas have been said to be pathognomic of FAP, but we have demonstrated that they also occur in AFAP and MAP. Given that some FAP microadenomas harbour somatic mutations at APC (Thirlwell, unpublished data), it is possible that they are the earliest detectable lesion, which has been 'initiated' by 'two hits' at APC (germline plus somatic mutation for (A)FAP or two somatic mutations for MAP). The absence of microadenomas in our patients suggests that their cells does not acquire 'two hits' at $A P C$ at an appreciably faster rate than the general population. The mechanism of tumour predisposition in the MCRA cases may therefore involve a stage of tumorigenesis that is post-initiation.

\section{$\beta$-Catenin expression is increased in MCRA tumours}

Relative to the 27 sporadic adenomas, the 57 MCRA tumours showed increased nuclear $\beta$-catenin expression ( $P=0.0087$, Wilcoxon test) (Figure 1). There was, however, no significant difference in nuclear $\beta$-catenin expression between the MCRA group's tumours and a set of 33 adenomas of similar size from six individuals with FAP $(P=0.09$, Wilcoxon test). Expression was not significantly different between MCRA and sporadic adenomas in the cytoplasm $(P=0.12$, Wilcoxon test) or at the membrane $(P=0.63$, Wilcoxon test) (Figure 2). Logistic regression analysis incorporating the three sites of $\beta$-catenin expression, adenoma size, degree of dysplasia and patient age found increased nuclear expression to be the only significant variable which distinguished MCRA and sporadic tumours $(P=0.017, \mathrm{OR}=0.22,95 \% \mathrm{CI} 0.065-0.76)$.

Unlike the other molecular analyses described above, $\beta$-catenin immunohistochemistry showed clear differences between MCRA and sporadic adenomas, with expression in MCRA tumours being similar to FAP. The greater Wnt activation in MCRA patients' tumours was too consistent to be readily explained by the inclusion of one or two individuals with cryptic germline $A P C$ or $M Y H$ mutations in the MCRA group. Allowing for the fact that colorectal microadenomas probably vary in their progression to macroscopic lesions (Crabtree et al, 2001), this finding raises the possibility that increased Wnt activation in MCRA leads microadenomas more rapidly or with greater probability to progress to macroadenomas. Studies to date (Lipton et $a l, 2003 \mathrm{~b}$ ) have not identified germline mutations in Wnt pathway genes in MCRA cases, but many such genes have not yet been screened.

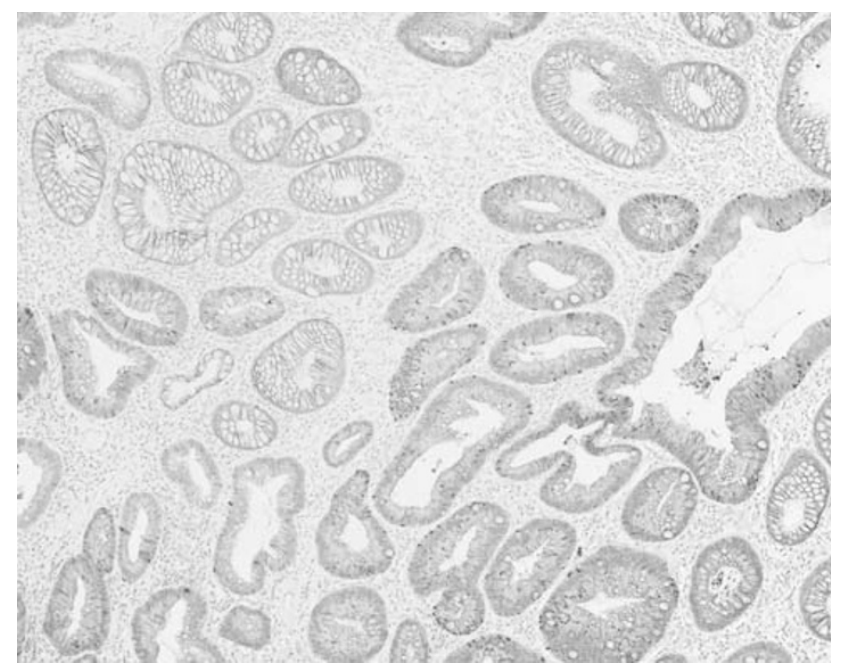

Figure $2 \beta$-Catenin immunohistochemistry in MCRA patient's adenoma. Multiple crypts with nuclear $\beta$-catenin expression are shown.

\section{CONCLUSION}

To date, Mendelian CRC syndromes can be categorised in two ways. First, the primary predisposition may be to polyps or to carcinoma. Second, the primary defect may be in either a 'gatekeeper gene' (such as APC,LKB1, SMAD4/MADH4 and $A L K 3 / B M P R 1 A$ ) or a caretaker/DNA repair gene (such as the MMR loci and MYH/MUTYH). The set of MCRA patients with no germline $A P C$ or $M Y H$ mutations is a priori likely to have a strong genetic predisposition, given the phenotypic similarity to MAP and AFAP. In our patients, multigenerational inheritance of multiple adenomas was not found, suggesting recessive or, perhaps, multilocus inheritance. Several possible explanations for the MCRA phenotype exist, and these patients may be a heterogeneous group. Nevertheless, our results have provided clues as to the reasons for the MCRA phenotype. We found somatic mutation frequencies and spectra in MCRA adenomas that were very similar to comparable sporadic lesions, distinct from AFAP and, especially, MAP. No microadenomas were found in the MCRA patients' colons. These results suggest that hypermutation of APC does not cause our patients' disease and strongly suggests that MAP is not a paradigm for the remaining MCRA patients. Our preferred model is that those few microadenomas, which do occur spontaneously in MCRA cases, have an increased chance of progressing to a macroadenoma. This notion is supported by our immunohistochemical analysis of $\beta$-catenin expression. If this is the case, it seems unlikely that the predisposition gene is a 'gatekeeper' type of tumour suppressor (since MCRA disease seems to be isolated or recessive) or a 'caretaker' (since we have found no evidence of a specific DNA repair defect). We tentatively suggest that our data fit best a modified 'landscaper' model of tumorigenesis in which the intra- or extracellular microenvironment of cells is altered to favour tumour progression.

\section{ACKNOWLEDGEMENTS}

We thank colleagues from the St Mark's Hospital Colorectal Cancer Unit for sample collection and from the Cancer Research UK London Research Institute (Equipment Park and Department of Histopathology) for assistance with genotyping and mutation detection. Emmanuel Volikos helped with sample collection and Andrew Silver kindly read the paper. 


\section{REFERENCES}

Al-Tassan N, Chmiel NH, Maynard J, Fleming N, Livingston AL, William GT, Hodges AK, Davies DR, David SS, Sampson JR Cheadle JP (2002) Inherited variants of MYH associated with somatic $\mathrm{G}: \mathrm{C} \rightarrow \mathrm{T}: \mathrm{A}$ mutations in colorectal tumors. Nat Genet 30: 227-232

Albuquerque C, Breukel C, van der Luijt R, Fidalgo P, Lage P, Slors FJ, Leitao CN, Fodde R, Smits R (2002) The 'just-right' signaling model: APC somatic mutations are selected based on a specific level of activation of the beta-catenin signaling cascade. Hum Mol Genet 11: 1549-1560

Crabtree M, Sieber OM, Lipton L, Hodgson SV, Lamlum H, Thomas HJ, Neale K, Phillips RK, Heinimann K, Tomlinson IP (2003) Refining the relation between 'first hits' and 'second hits' at the APC locus: the 'loose fit' model and evidence for differences in somatic mutation spectra among patients. Oncogene 22: $4257-4265$

Crabtree MD, Tomlinson IP, Talbot IC, Phillips RK (2001) Variability in the severity of colonic disease in familial adenomatous polyposis results from differences in tumour initiation rather than progression and depends relatively little on patient age. Gut 49: $540-543$

Groves C, Lamlum H, Crabtree M, Williamson J, Taylor C, Bass S, CuthbertHeavens D, Hodgson S, Phillips R, Tomlinson I (2002) Mutation cluster region, association between germline and somatic mutations and genotype-phenotype correlation in upper gastrointestinal familial adenomatous polyposis. Am J Pathol 160: 2055-2061

Lamlum H, Al Tassan N, Jaeger E, Frayling I, Sieber O, Reza FB, Eckert M, Rowan A, Barclay E, Atkin W, Williams C, Gilbert J, Cheadle J, Bell J, Houlston R, Bodmer W, Sampson J, Tomlinson I (2000) Germline APC variants in patients with multiple colorectal adenomas, with evidence for the particular importance of E1317Q. Hum Mol Genet 9: 2215-2221

Lamlum H, Ilyas M, Rowan A, Clark S, Johnson V, Bell J, Frayling I, Efstathiou J, Pack K, Payne S, Roylance R, Gorman P, Sheer D, Neale K, Phillips R, Talbot I, Bodmer W, Tomlinson I (1999) The type of somatic mutation at APC in familial adenomatous polyposis is determined by the site of the germline mutation: a new facet to Knudson's 'two-hit' hypothesis. Nat Med 5: 1071-1075

Laurent-Puig P, Beroud C, Soussi T (1998) APC gene: database of germline and somatic mutations in human tumors and cell lines. Nucleic Acids Res 26: $269-270$
Lipton L, Halford SE, Johnson V, Novelli MR, Jones A, Cummings C, Barclay E, Sieber O, Sadat A, Bisgaard ML, Hodgson SV, Aaltonen LA, Thomas HJ, Tomlinson IP (2003a) Carcinogenesis in MYH-associated polyposis follows a distinct genetic pathway. Cancer Res 63: 7595-7599

Lipton L, Sieber OM, Thomas HJ, Hodgson SV, Tomlinson IP, WoodfordRichens K (2003b) Germline mutations in the TGF-beta and Wnt signalling pathways are a rare cause of the "multiple" adenoma phenotype. J Med Genet 40: e35

Sampson JR, Dolwani S, Jones S, Eccles D, Ellis A, Evans DG, Frayling I, Jordan S, Maher ER, Mak T, Maynard J, Pigatto F, Shaw J, Cheadle JP (2003) Autosomal recessive colorectal adenomatous polyposis due to inherited mutations of MYH. Lancet 362: 39-41

Sieber O, Segditsas S, Knudsen A, Zhang J, Luz J, Rowan A, Spain S, Thirlwell C, Howarth K, Jaeger E, Robinson J, Volikos E, Silver A, Kelly G, Aretz S, Frayling I, Hutter P, Dunlop M, Guenther T, Neale K, Phillips R, Heinimann K, Tomlinson I (2006) Disease severity and genetic pathways in attenuated familial adenomatous polyposis vary greatly, but depend on the site of the germline mutation. Gut 55(10): 1440-1448

Sieber OM, Lipton L, Crabtree M, Heinimann K, Fidalgo P, Phillips RK, Bisgaard ML, Orntoft TF, Aaltonen LA, Hodgson SV, Thomas HJ, Tomlinson IP (2003) Multiple colorectal adenomas, classic adenomatous polyposis, and germ-line mutations in MYH. N Engl J Med 348: 791 - 799

Spirio L, Green J, Robertson J, Robertson M, Otterud B, Sheldon J, Howse E, Green R, Groden J, White R, Leppert M (1999) The identical $5^{\prime}$ splice-site acceptor mutation in five attenuated APC families from Newfoundland demonstrates a founder effect. Hum Genet 105: 388-398

Woodford-Richens KL, Rowan AJ, Gorman P, Halford S, Bicknell DC, Wasan HS, Roylance RR, Bodmer WF, Tomlinson IP (2001) SMAD4 mutations in colorectal cancer probably occur before chromosomal instability, but after divergence of the microsatellite instability pathway. Proc Natl Acad Sci USA 98: 9719-9723

Wu Y, Berends MJ, Mensink RG, Kempinga C, Sijmons RH, van Der Zee AG, Hollema H, Kleibeuker JH, Buys CH, Hofstra RM (1999) Association of hereditary nonpolyposis colorectal cancer-related tumors displaying low microsatellite instability with MSH6 germline mutations. Am J Hum Genet 65: 1291 - 1298 